\title{
Developments at ISOL facilities: from ISOLDE towards EURISOL
}

\author{
Y. Blumenfeld ${ }^{\mathrm{i}}$ \\ CERN/ISOLDE, 1211 Geneva 23, Switzerland
}

The ISOLDE facility at CERN has been a world-leading ISOL facility for many years and since the inception of the REX post-accelerator has delivered accelerated beams of over 50 isotopes of elements as light as Li and as heavy as Rn. The physics program will be illustrated through a few representative examples including mass measurements, laser spectroscopy, Coulomb excitation and transfer reactions. An upgrade called HIE-ISOLDE, which aims to increase the radioactive beam energy and to handle the higher intensities delivered by the CERN injector system will be described.

In a longer term, the European long range plan for Nuclear Physics calls for the construction of the "ultimate" ISOL facility: EURISOL. Over the past 5 years, twenty European laboratories have participated in the Design Study of EURISOL. The general layout of the facility will be described and the tests of several prototypes of the most challenging parts of EURISOL, including accelerator cryomodules and high power targets will be reported.

We acknowledge the financial support of the European Community under the FP6 "Research Infrastructure Action - Structuring the European Research Area" EURISOL DS Project Contract no. 515768 RIDS. The EC is not liable for any use that can be made of the information contained herein.

'On leave from IPN Orsay, France 